\title{
Symmetry based 3D Reconstruction of Repeated Cylinders
}

\author{
Adersh Miglani \\ Adersh.Miglani@gmail.com
}

\author{
Sumantra Dutta Roy \\ sumantra@ee.iitd.ac.in
}

\author{
Santanu Chaudhury \\ santanuc@ee.iitd.ac.in
}

\author{
J B Srivastava \\ jbsrivas@gmail.com
}

\begin{abstract}
First, we describe how $360^{\circ}$-rotational symmetry may be used for three dimensional reconstruction of repeated cylinders from a single perspective image. In our experiments, we consider translational and affine repetition of cylinders with vertical and random orientations. Later, we create a virtual camera configuration for retrieving pose and location of repeated cylinders. The combination of $360^{\circ}$-rotational symmetry and camera center is used to identify two orthogonal planes called axis plane and orthogonal axis plane. These two planes are the basis for the proposed reconstruction framework and virtual camera configuration. Furthermore, we discuss possible extension of our method in vision tasks based on motion analysis.
\end{abstract}

\section{INTRODUCTION}

Computational symmetry and group theory has been extensively studied in computer vision [1]. It is widely used for image segmentation and retrieving three dimensional structure and pose and computing structure from motion [2][3][4][5]. Here, we use rotational symmetry for 3D reconstruction of repeated cylinders with random orientations from single perspective image. These cylinders may have translational and affine repetitions. Since a cylinder has $360^{\circ}$-rotational symmetry around its axis, it is convenient to use vanishing points based visual metrology techniques.

Single view metrology techniques are mostly used for retrieving metric measurements and calibrating cameras [6][7][8][9]. These techniques use homology, a plane projective transformation, for scenes containing parallel lines and parallel planes [10][6][7]. In the proposed method, we blend and develop previous work on $(i)$ vanishing points based reconstruction methods; (ii) symmetry of individual 3D object; (iii) configuration of repeated objects.

In this paper, we consider a prespective image of randomly placed cylinders on the ground plane. Any one cylinder is chosen as principal cylinder and rest are called auxiliary cylinders. The $360^{\circ}$-rotational symmetry and location of camera projection center are used in finding two orthogonal planes. First, axis plane passes through camera center and axis of cylinder. It bisects cylinder in Euclidean space and image plane too, if we ignore the lens distortion. Second, orthogonal axis plane passes through cylinder's axis and is orthogonal to axis plane.

These two planes are the basis for 3D reconstruction framework and creating a virtual camera configuration, an alternate framework, for retrieving 3D-box (pose and location) of auxiliary cylinder with respect to principal cylinder. Furthermore, proposed framework can be extended for vision tasks involving multiple views such as motion analysis - structure from motion, motion segmentation and tracking.

Primarily, homology is used to find the distance between two parallel planes [6][10]. A set of three orthogonal pairs of parallel planes are required for retrieving 3D coordinates of a point. This is a three homology configuration. Since, a cylinder has $360^{\circ}$-rotational symmetry around its axis, it requires only two homology to find 3D coordinates of a point on the top surface of a vertical cylinder. The 3D coordinates of the point give height and diameter. Similar techniques are used to find the measurements and position of arbitrarily placed auxiliary cylinder with respect to the principal cylinder.

Section II describes $360^{\circ}$-rotational symmetry and two important orthogonal planes for a cylinder. A framework for computing measurement and location of principal and auxiliary cylinders is developed in section III. Detailed discussion on the virtual camera configuration for computing 3D-box of repeated cylinders is described in section IV. Results and conclusion with future work are discussed in Section V and VI, respectively.

\section{Symmetry in the GeOMEtRy OF A CYLINDER}

The chosen world coordinate system is shown in figure (1). Every cylinder has such coordinate system. However, measurements and location of all auxiliary cylinders are computed with respect to principal cylinder. Particularly, this coordinate system is useful in understanding rotational symmetry, finding two orthogonal planes, creating virtual camera configuration, as discussed in section IV, and computing point correspondences from image. 


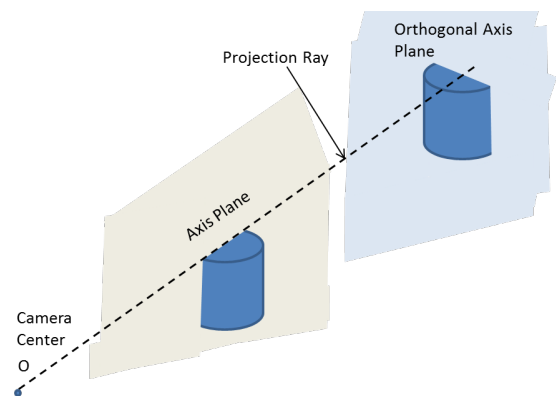

Fig. 2. Axis Plane and Orthogonal Axis Plane

\section{A. Rotational Symmetry and Projection Center}

All the projectors from camera center to a cylinder's axis form a plane. This is called an axis plane. The image of axis plane or image of cylinder's axis is a line segment that bisects the image of cylinder, if we ignore the lens distortion. Consequently, there is reflection symmetry in the image of every cylinder. This plane is identified due to $360^{\circ}$-rotational symmetry in the shape of cylinder. Another important plane is orthogonal axis plane. It passes through cylinder's axis and is orthogonal to axis plane. These two planes are shown in figure (2).

"If a cylinder is rotated around its axis or is moved in world space such that the perpendicular distance of orthogonal axis plane from camera center is kept constant, the size and shape of its image will remain same".

This is an important conclusion which relates the motion of cylinder and its $360^{\circ}$-rotational symmetry.

\section{B. Point Correspondences}

The axis plane and orthogonal axis plane are used to find corresponding points for each cylinder as shown in figure (1). Points $m_{i}$ are image of points $M_{i}$, where $i=0, \ldots, 8$. Image points $m_{1}, m_{3}, m_{5}$, and $m_{7}$ lie on orthogonal axis plane. The lines $\left\langle m_{1}, m_{5}\right\rangle$ and $\left\langle m_{3}, m_{7}\right\rangle$ are side-edges of image of cylinder and can be computed using edge detection techniques. Due to reflection symmetry, $m_{8}$ is mid point of $m_{1}$ and $m_{3}$ and image of visible circular surface is ellipse, if lens distortion is ignored. The line passing through $m_{8}$ and orthogonal to line $\left\langle m_{1}, m_{3}\right\rangle$ intersects the conic at $m_{2}$ and $m_{4}$. We can use Bookstein's method for conic fitting [11]. Point $M_{8}$ is mid point of $M_{2}$ and $M_{4}$. Due to perspective distortion $m_{8}$ would not be mid point of $m_{2}$ and $m_{4}$. Point $m_{6}$ can be computed in the similar manner.

\section{MEASUREMENT AND LOCATION OF CYLINDERS}

\section{A. Measurement of Principal Cylinder}

The vanishing points of $X$ and $Z$ axes are computed as follows:

$v_{x}=\left(m_{1} \times m_{3}\right) \times\left(m_{5} \times m_{7}\right), v_{z}=\left(m_{3} \times m_{7}\right) \times\left(m_{1} \times m_{5}\right)$

Since $M_{8}$ is mid point of $M_{2}$ and $M_{4}, 1 D$ homography is used to compute the vanishing point of $Y$ axis, $v_{y}$ [10]. Point $v_{0}$ is image of world origin.

$$
v_{0}=\left(m_{6} \times v_{x}\right) \times\left(m_{7} \times v_{y}\right)
$$

To find measurements of principal cylinder, 3D coordinates of $M_{1}=\left(X_{1}, Y_{1}, Z_{1}\right)$ should be computed. Point $M_{5}=$ $\left(X_{1}, Y_{1}, 0\right)$ is projection of $M_{1}$ on $X Y$-plane. The camera projection matrix can be chosen as $P=\left(\begin{array}{llll}v_{x} & v_{y} & v_{z} & v_{0}\end{array}\right)$ [10][7]. The projection of $M_{5}$ in terms of $P$ is written as follows

$$
\lambda_{5} m_{5}=X_{1} v_{x}+Y_{1} v_{y}+v_{0}
$$

Taking scalar product of above equation with $l_{x y}$ yields $\lambda_{5}$.

$$
\lambda_{5}=\frac{\left(v_{0} \cdot l_{x y}\right)}{\left(m_{5} \cdot l_{x y}\right)}
$$

Similarly, $m_{1}$ can be expressed in terms of $m_{5}$.

$$
\lambda_{1} m_{1}=X_{1} v_{x}+Y_{1} v_{y}+Z_{1} v_{z}+v_{0}=\lambda_{5} m_{5}+Z_{1} v_{z}
$$

Taking vector product of above equation with $m_{1}$ yields $Z_{1}$.

$$
Z_{1}=-\lambda_{5} \frac{\left\|m_{5} \times m_{1}\right\|}{\left\|v_{z} \times m_{1}\right\|}
$$

Similarly, after taking scalar product of equation for $m_{5}$ with $l_{z x}$ and $l_{y z}$, we get $Y_{1}$ and $X_{1}$, respectively.

$$
Y_{1}=\frac{\lambda_{5}\left(m_{5} \cdot l_{z x}\right)-\left(v_{0} \cdot l_{z x}\right)}{\left(v_{y} \cdot l_{z x}\right)}, X_{1}=\frac{\lambda_{5}\left(m_{5} \cdot l_{y z}\right)-\left(v_{0} \cdot l_{y z}\right)}{\left(v_{x} \cdot l_{y z}\right)}
$$

We assumed that principal cylinder is vertically oriented, though not necessary, and its height $H$ and diameter $D$ are known. The $X_{1}, Y_{1}$ and $Z_{1}$ are affine measurements. The constants $\alpha_{x}=\frac{X_{1}}{D}, \alpha_{y}=\frac{2 Y_{1}}{D}$ and $\alpha_{z}=\frac{Z_{1}}{H}$ are fixed for the chosen world coordinate system [7]. So, the position of camera center, $T_{C}=\left(T_{C X} T_{C Y} T_{C Z}\right)^{T}$, is computed using vanishing points and these three constants [7][10].

\section{B. Measurements and Pose of Auxiliary Cylinders}

Position of auxiliary cylinder is computed with respect to principal cylinder. The key idea is not to use vanishing points of auxiliary cylinder. In this context, two different configurations are considered.

1) Vertical Orientation: Vertically oriented auxiliary cylinder may have translational or affine repetition. To find the measurement and location of auxiliary cylinder, 3D coordinates of $M_{1}^{\prime}=\left(X_{1}^{\prime}, Y_{1}^{\prime}, Z_{1}^{\prime}\right)$, and $M_{3}^{\prime}=\left(X_{3}^{\prime}, Y_{3}^{\prime}, Z_{1}^{\prime}\right)$ should be computed. $M_{5}^{\prime}=\left(X_{1}^{\prime}, Y_{1}^{\prime}, 0\right)$ and $M_{7}^{\prime}=\left(X_{3}^{\prime}, Y_{3}^{\prime}, 0\right)$ are projection of $M_{1}^{\prime}$ and $M_{3}^{\prime}$ on the $X Y$-plane, respectively.

$$
\lambda_{5}^{\prime} m_{5}^{\prime}=X_{1}^{\prime} v_{x}+Y_{1}^{\prime} v_{y}+v_{0} \Rightarrow \lambda_{5}^{\prime}=\frac{\left(v_{0} \cdot l_{x y}\right)}{\left(m_{5}^{\prime} \cdot l_{x y}\right)}
$$

Image point $m_{1}^{\prime}$ can be written in terms of $m_{5}^{\prime}$,

$$
\lambda_{1}^{\prime} m_{1}^{\prime}=Z_{1}^{\prime} v_{z}+\lambda_{5}^{\prime} m_{5}^{\prime}
$$

Taking scalar product of above equation with $m_{1}^{\prime}$ yields $Z_{1}^{\prime}$.

$$
Z_{1}^{\prime}=-\lambda_{5}^{\prime} \frac{\left\|m_{5}^{\prime} \times m_{1}^{\prime}\right\|}{\left\|v_{z} \times m_{1}^{\prime}\right\|}
$$


Using $\lambda_{5}^{\prime}$ in the equation for $m_{5}^{\prime}$, we get expressions for $X_{1}^{\prime}$ and $Y_{1}^{\prime}$,

$$
X_{1}^{\prime}=\frac{\lambda_{5}^{\prime}\left(m_{5}^{\prime} \cdot l_{y z}\right)-\left(v_{0} \cdot l_{y z}\right)}{\left(v_{x} \cdot l_{y z}\right)}, Y_{1}^{\prime}=\frac{\lambda_{5}^{\prime}\left(m_{5}^{\prime} \cdot l_{z x}\right)-\left(v_{0} \cdot l_{z x}\right)}{\left(v_{y} \cdot l_{z x}\right)}
$$

In the similar fashion, we can solve equations for $m_{3}^{\prime}$ and $m_{7}^{\prime}$ and get expressions for $X_{3}^{\prime}$ and $Y_{3}^{\prime}$.

$$
\begin{gathered}
\lambda_{7}^{\prime} m_{7}^{\prime}=X_{3}^{\prime} v_{x}+Y_{3}^{\prime} v_{y}+v_{0} \Rightarrow \lambda_{7}^{\prime}=\frac{\left(v_{0} \cdot l_{x y}\right)}{\left(m_{7}^{\prime} \cdot l_{x y}\right)} \\
Y_{3}^{\prime}=\frac{\lambda_{7}^{\prime}\left(m_{7}^{\prime} \cdot l_{z x}\right)-\left(v_{0} \cdot l_{z x}\right)}{\left(v_{z} \cdot l_{z x}\right)}, X_{3}^{\prime}=\frac{\lambda_{7}^{\prime}\left(m_{7}^{\prime} \cdot l_{y z}\right)-\left(v_{0} \cdot l_{y z}\right)}{\left(v_{x} \cdot l_{y z}\right)}
\end{gathered}
$$

2) Random Orientation: In this configuration cylinders are placed horizontally, as shown in figure (6). It is another case of affine repetition. The $X Y$-plane is a tangent plane to the auxiliary cylinder and touches in a line segment. One end point of this segment is $M_{9}^{\prime}=\left(X_{10}^{\prime}, Y_{10}^{\prime}, 0\right)$. The diameter through $M_{9}^{\prime}$ intersects the conic at $M_{10}^{\prime}=\left(X_{10}^{\prime}, Y_{10}^{\prime}, Z_{10}^{\prime}\right)$. Another hypothetical plane, which is parallel to $X Y$-plane, touches cylinder in a line segment $\left\langle M_{10}^{\prime}, M_{11}^{\prime}\right\rangle$. The coordinates of point $M_{11}^{\prime}$ are $\left(X_{11}^{\prime}, Y_{11}^{\prime}, Z_{10}^{\prime}\right)$. To find the measurement and location of auxiliary cylinder, 3D coordinates of $M_{10}^{\prime}$ and $M_{11}^{\prime}$ should be computed.

The line $v_{z} \times m_{8}^{\prime}$ intersects the visible conic at points $m_{9}^{\prime}$ and $m_{10}^{\prime}$. In the similar fashion, image coordinates of $m_{11}^{\prime}$ can be computed. Once, image coordinates of $m_{9}^{\prime}, m_{10}^{\prime}$ and $m_{11}^{\prime}$ are computed, the expressions for $\lambda_{10}, X_{10}^{\prime}, Y_{10}^{\prime}$ and $Z_{10}^{\prime}$ are worked out as before,

$$
\begin{gathered}
\lambda_{9}^{\prime} m_{9}^{\prime}=X_{10}^{\prime} v_{x}+Y_{10}^{\prime} v_{y}+v_{0} \Rightarrow \lambda_{9}^{\prime}=\frac{\left(v_{0} \cdot l_{x y}\right)}{\left(m_{9}^{\prime} \cdot l_{x y}\right)} \\
X_{10}^{\prime}=\frac{\lambda_{9}^{\prime}\left(m_{9}^{\prime} \cdot l_{y z}\right)-\left(v_{0} \cdot l_{y z}\right)}{\left(v_{x} \cdot l_{y z}\right)}, Y_{10}^{\prime}=\frac{\lambda_{9}^{\prime}\left(m_{9}^{\prime} \cdot l_{z x}\right)-\left(v_{0} \cdot l_{z x}\right)}{\left(v_{y} \cdot l_{z x}\right)} \\
\lambda_{10}^{\prime} m_{10}^{\prime}=Z_{10}^{\prime} v_{z}+\lambda_{9}^{\prime} m_{9}^{\prime} \Rightarrow Z_{10}^{\prime}=-\lambda_{9}^{\prime} \frac{\left\|m_{9}^{\prime} \times m_{10}^{\prime}\right\|}{\left\|v_{z} \times m_{10}^{\prime}\right\|}
\end{gathered}
$$

We need to use $Z_{10}^{\prime}$ to compute expression for $X_{11}^{\prime}$ and $Y_{11}^{\prime}$.

$$
\begin{aligned}
\lambda_{11}^{\prime} m_{11}^{\prime} & =X_{11}^{\prime} v_{x}+Y_{11}^{\prime} v_{y}+Z_{10}^{\prime} v_{z}+v_{0} \\
\lambda_{11}^{\prime} & =\frac{Z_{10}^{\prime}\left(v_{z} \cdot l_{x y}\right)+\left(v_{0} \cdot l_{x y}\right)}{\left(m_{11}^{\prime} \cdot l_{x y}\right)} \\
X_{11}^{\prime} & =\frac{\lambda_{11}^{\prime}\left(m_{11}^{\prime} \cdot l_{y z}\right)-\left(v_{0} \cdot l_{y z}\right)}{\left(v_{x} \cdot l_{y z}\right)} \\
Y_{11}^{\prime} & =\frac{\lambda_{11}^{\prime}\left(m_{11}^{\prime} \cdot l_{z x}\right)-\left(v_{0} \cdot l_{z x}\right)}{\left(v_{y} \cdot l_{z x}\right)}
\end{aligned}
$$

\section{Virtual CAmera Configuration}

The proposed virtual camera configuration framework corroborates the previous discussion and provide an alternate method to compute 3D-box of auxiliary cylinders. A single perspective image of repeated cylinders can be considered as multiple images of one cylinder. This is called isometry property. Axis plane and orthogonal axis plane are dependent on camera center. Virtual camera for auxiliary cylinder is placed such that virtual axis plane and virtual orthogonal axis

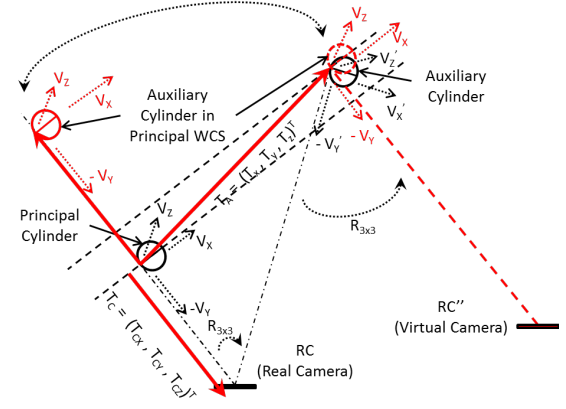

Fig. 3. Virtual Camera Configuration

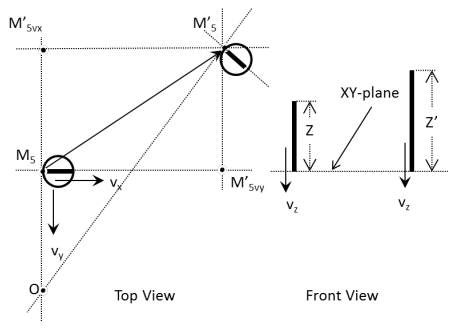

Fig. 4. Location of Affinely Repeated Cylinder

plane are parallel to axis plane and orthogonal axis plane of principal cylinder, respectively, as shown in figure (3). This configuration of planes can be used to find the position of auxiliary cylinder with respect to principal cylinder.

\section{A. Vertical Orientation}

1) Translational Repetition: The position of auxiliary cylinder with respect to principal cylinder is represented by 3vector $T^{\prime}=\left(\begin{array}{lll}X^{\prime} & Y^{\prime} & 0\end{array}\right)^{T}$, as shown in the figure (4) (top view). If $M_{5}=\left(\begin{array}{lll}X_{5} & Y_{5} & 0\end{array}\right)$ and $M_{5}^{\prime}=\left(\begin{array}{lll}X_{5}^{\prime} & Y_{5}^{\prime} & 0\end{array}\right)$ is a pair of corresponding point, length of line segments $\left\langle M_{5}^{\prime}, M_{5 v x}^{\prime}\right\rangle$ and $\left\langle M_{5}^{\prime}, M_{5 v y}^{\prime}\right\rangle$ are $X$ and $Y$ coordinates of vector $T^{\prime}$. Points $M_{5 v x}^{\prime}=\left(\begin{array}{lll}X_{5}+X^{\prime} & Y_{5} & 0\end{array}\right)$ and $M_{5 v y}^{\prime}=$ $\left(\begin{array}{lll}X_{5} & Y_{5}+Y^{\prime} & 0\end{array}\right)$ are projection of point $M_{5}^{\prime}$ on the planes which pass through point $M_{5}$ and are parallel to $Y Z$ and $Z X$ planes, respectively.

$$
\begin{aligned}
& m_{5 v x}^{\prime}=\left(m_{5} \times v_{y}\right) \times\left(m_{5}^{\prime} \times v_{x}\right) \\
& m_{5 v y}^{\prime}=\left(m_{5} \times v_{x}\right) \times\left(m_{5}^{\prime} \times v_{y}\right)
\end{aligned}
$$

Once image coordinates $m_{5 v x}^{\prime}$ and $m_{5 v y}^{\prime}$ are computed, $X^{\prime}$ and $Y^{\prime}$ can be computed by using similar calculus as discussed in section III.

2) Affine Repetition: In case of affine repetition, 3-vector $T^{\prime}=\left(\begin{array}{lll}X^{\prime} & Y^{\prime} & Z^{\prime}\end{array}\right)^{T}$ has non-zero $Z$ coordinate, as shown in figure (4) (Front View). The $X^{\prime}$ and $Y^{\prime}$ coordinates are computed same as for translational repetition and expression for $Z^{\prime}$ is

$$
Z^{\prime}=\frac{\left\|\lambda_{1}^{\prime}\left(m_{1}^{\prime} \times l_{x y}\right)-\left(v_{0} \times l_{x y}\right)\right\|}{\left\|v_{z} \times l_{x y}\right\|}
$$

where

$$
\lambda_{1}^{\prime}=\frac{\left\|\left(X_{5}+X^{\prime}\right)\left(v_{x} \times l_{y z}\right)+\left(v_{0} \times l_{y z}\right)\right\|}{\left\|m_{1}^{\prime} \times l_{y z}\right\|}
$$




\section{B. Random orientation}

In this case, pose along with position is required for computing $3 D$ box of auxiliary cylinder with respect to principal cylinder. The vector $T^{\prime}$ is computed as per section III-B2. Pose, which is rotation transformation, is computed using set of vanishing points of both cylinders. Geometry is shown in the figure (3).

$$
\left(\begin{array}{lll}
v_{x}^{\prime} & v_{y}^{\prime} & v_{z}^{\prime}
\end{array}\right)=R_{3 \times 3}\left(\begin{array}{lll}
v_{x} & v_{y} & v_{z}
\end{array}\right)
$$

Given metric measurements of principal cylinder (distances along $X, Y$ and $Z$ axes), $R_{3 \times 3}$ can be computed [9][8].

\section{EXPERIMENTAL RESULTS}

In our experiment, we considered two real images (figure (5) and (6)). Table I and II display the measurements (centimeter) of cylinders computed using methods discussed in section III-B1 and III-B2, respectively. The deviation of experimental measurements from scene measurements is acceptable. Those can be further improved by using efficient image processing techniques for finding point correspondences and computing vanishing points. There is a possibility of further improvement by employing uncertainly analysis as discussed in [7].

TABLE I

EXPERIMENTAL RESULTS FOR FIGURE (5)

\begin{tabular}{lccrrr}
\hline \hline Cylinder & Orientation & Type & Scene & Experiment & Error \\
\hline C1 & \multirow{2}{*}{ Vertical } & Diameter & 3.1 & 2.8 & -0.3 \\
& & Height & 5.0 & 4.5 & -0.5 \\
C2 & \multirow{2}{*}{ Vertical } & Diameter & 2.6 & 2.9 & 0.3 \\
& & Height & 4.1 & 4.6 & 0.5 \\
& \multirow{3}{*}{ Certical } & Diameter & 2.6 & 2.5 & -0.1 \\
& & Height & 6.1 & 5.8 & -0.3 \\
\hline
\end{tabular}

TABLE II

EXPERIMENTAL RESULTS FOR FIGURE (6)

\begin{tabular}{lccrrr}
\hline \hline Cylinder & Orientation & Type & Scene & Experiment & Error \\
\hline \multirow{2}{*}{ C1 } & \multirow{2}{*}{ Vertical } & Diameter & 3.1 & 3.3 & 0.2 \\
& & Height & 5.0 & 5.3 & 0.3 \\
C2 & \multirow{2}{*}{ Horizontal } & Diameter & 2.6 & 2.6 & 0.0 \\
& & Height & 4.1 & 5.2 & 1.1 \\
C3 & \multirow{2}{*}{ Horizontal } & Diameter & 2.6 & 2.8 & 0.2 \\
& & Height & 6.1 & 7.9 & 1.8 \\
\hline
\end{tabular}

\section{CONCLUSION}

We extended previous work on single view metrology and effectively utilized rotational symmetry for three dimensional reconstruction of repeated objects. The proposed method showed acceptable results with the scope of further improvements. The $360^{\circ}$-rotational symmetry based virtual camera configuration framework can be further extended for view synthesis, motion analysis - segmentation and tracking. As well, proposed framework can be extended for vision tasks related to individual or repeated objects with reflection symmetry, uniform finite rotational symmetry and skewed symmetry.

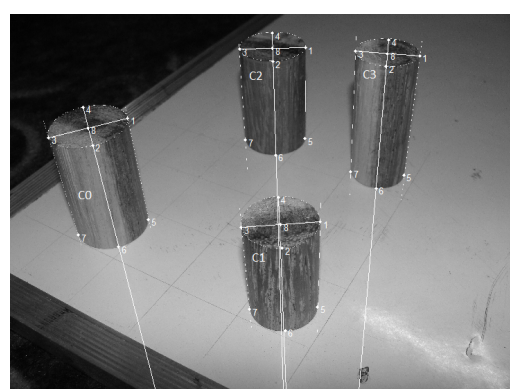

Fig. 5. Cylinders with Vertical Orientations

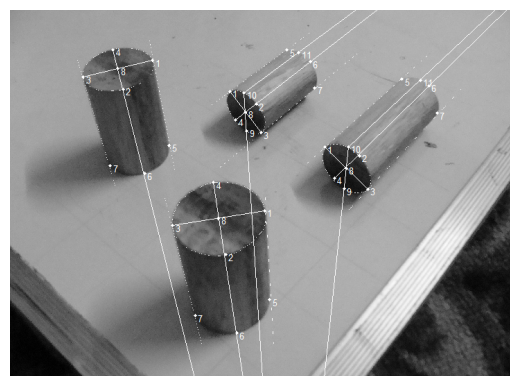

Fig. 6. Cylinders with Random Orientation

\section{ACKNOWLEDGMENT}

This work has been supported under the research grant, towards the setting up of the 'Program on Autonomous Robotics', from the Board of Research in Nuclear Sciences (BRNS), India.

\section{REFERENCES}

[1] Y. Liu, H. Hel-Or, C. S. Kaplan, and L. J. V. Gool, "Computational symmetry in computer vision and computer graphics," Foundations and Trends in Computer Graphics and Vision, vol. 5, no. 1-2, pp. 1-195, 2010.

[2] A. Y. Yang, S. Rao, K. Huang, W. Hong, and Y. Ma, "Geometric segmentation of perspective images based on symmetry groups," in In Proceedings of the International Conference on Computer Vision, 2003, pp. 1251-1258.

[3] W. Hong, "On symmetry and multiple-view geometry: Structure, pose, and calibration from a single image," IJCV, vol. 60, 2004.

[4] J. Liu, J. L. Mundy, and A. Zisserman, "Grouping and structure recovery for images of objects with finite rotational symmetry," in Asian Conference on Computer Vision, vol. I, 1995, pp. 379-382.

[5] M. Pollefeys, "Discovering and exploiting 3d symmetries in structure from motion," in Proceedings of the 2012 IEEE Conference on Computer Vision and Pattern Recognition (CVPR), ser. CVPR '12. Washington, DC, USA: IEEE Computer Society, 2012, pp. 1514-1521.

[6] A. Criminisi, I. Reid, and A. Zisserman, "Single view metrology," Int. J. Comput. Vision, vol. 40, no. 2, pp. 123-148, Nov. 2000.

[7] A. Criminisi, "Accurate visual metrology from single and multiple uncalibrated images," Ph.D. dissertation, University of Oxford, Dept. Engineering Science, 1999, d.Phil. thesis.

[8] B. Caprile and V. Torre, "Using vanishing points for camera calibration," International Journal of Computer Vision, vol. 4, no. 2, pp. 127-139, Mar. 1990. [Online]. Available: http://dx.doi.org/10.1007/bf00127813

[9] E. Guillou, D. Meneveaux, E. Maisel, and K. Bouatouch, "Using vanishing points for camera calibration and coarse $3 \mathrm{~d}$ reconstruction from a single image," The Visual Computer, vol. 16, no. 7, pp. 396-410, 2000. [Online]. Available: http://dx.doi.org/10.1007/PL00013394

[10] R. I. Hartley and A. Zisserman, Multiple View Geometry in Computer Vision, 2nd ed. Cambridge University Press, ISBN: 0521540518, 2004.

[11] F. L. Bookstein, "Fitting conic sections to scattered data," Computer Graphics and Image Processing, vol. 9, no. 1, pp. 56 - 71, 1979. 